\title{
Subpixel Edge Localization Based on the Bresenham Algorithm
}

\author{
Sun Weifang ${ }^{\mathrm{a}}$, Chen Binqiang ${ }^{\mathrm{b}}$ and Luo Qi ${ }^{*}$ \\ School of Aerospace Engineering, Xiamen University \\ No. 422, Siming South Road, Xiamen, Fujian, China. 361005 \\ a vincent_suen@126.com, ${ }^{b}$ cbq@xmu.edu.cn, ${ }^{c}$ xmuvicky@126.com
}

\begin{abstract}
Keywords: Subpixel edge localization. Bresenham algorithm. Photogrammetry. non-contact measurement

Abstract. Precise subpixel edge localization is significant for photogrammetry. In this paper, a new subpixel edge localization and interpolation of still image based on the Bresenham algorithm is proposed. Parameters fitting method is adopted to acquire the subpixel image brim point. After the localization, contiguous pixel along the gradient direction is convenience get according to the Bresenham algorithm. The indexable insert experiment shows a satisfactory result which means a potential good measurement precision.
\end{abstract}

\section{Introduction}

A number of non-contact inspection technologies were developed for increasing the accuracy of measurement during the manufacturing process. As the significant technology of image analysis and processing, edge localization technology has important value in measurement, industrial production, military target reconnaissance, agriculture, ocean etc[1-4]. Many algorithms are proposed for the edge detection, such as Sobel algorithm, Prewitt algorithm, Laplacian algorithm and Canny algorithm. Subpixel edge localization and high precision calibration are the main methods to the accuracy improvement.

Presented research for edge localization can be classified into three types: moment method, interpolation method and fitting method. Y. Shan and G. W. Boon generalizes the edge model to one containing a local blurring factor, and gives finite closed-form solutions for edge location with sub-pixel accuracy[5]. Based on a B-spline interpolation of the initial signal and of an optimized derivative filte, F. Truchetet, F. Nicolier and O. Laligant proposed An algorithm for edge detection and localization with subpixel resolution[6]. Jian Ye, Gongkang Fu and Upendra P. Poudel proposed a high-accuracy edge detection algorithm based on Gaussian edge model and least-squared-error implementation[7].

Based on parameters fitting method and Bresenham algorithm, this paper proposes a simplified method for detecting subpixel edge. The finally experiment result shows a satisfactory result which means a potential good measurement precision and good fault tolerance.

\section{Subpixel edge localization}

The Charge-Coupled Device (CCD) has been the dominant technology for visible photon detection and image capture over the past decades due to its ability to perform very efficiently and uniformly over large areas[8]. CCD devices used for photons detection are organized as arrays of photodetectors that deliver an electrical signal related to the amount of photons that fall on the pixel surface during the integration time. Fixed integration time and pixel area make the output signal is only related to the light intensity distribution. The output gray scale value can be described as:

$$
f(i, j)=\int_{i-0.5}^{i+0.5} \int_{j-0.5}^{j+0.5} g(x, y) d x d y
$$

where, $g(x, y)$ stands for the light intensity distribution of continue figure. Equ. 1 shows that gray scale value $f(i, j)$ reflects the area comprehensive light intensity distribution of the area. This method is called the square aperture sampling theorem[9]. Sampling result is a gray scale value dispersion matrix. 
The mutational sharp surface gray scale value in physical space becomes gradual because of the convolution effect of optical components and optical diffraction. Edges of still image contain a great amount of high-frequency information, it represented as a gray scale value distribution in image. Kris Jensen and Dimitris Anastassiou point out that the edge change followed Gaussian distribution, the maximum gray level difference as well as the vertex of Gaussian curve is its real edge in physical space[10]. The Gaussian curve can be represented as:

$$
y=\frac{1}{\sqrt{2 \pi \sigma}} \exp \left[\frac{-(x-\mu)^{2}}{2 \sigma^{2}}\right]
$$

where, $\mu$ is the mean value, $\sigma$ is the standard deviation.

For the convenience of calculation, Equ.2 can be simplified as:

$$
\ln y=\frac{-(x-\mu)^{2}}{2 \sigma^{2}}+\ln \frac{1}{\sqrt{2 \pi \sigma}}
$$

Let $y^{*}=\ln y$ Equ. 3 can be represented as:

$$
y^{*}=A x^{2}+B x+C
$$

According to the square aperture sampling theorem, the pixel gray scale value difference is:

$$
y^{*}(n)=\int_{n-0.5}^{n+0.5}\left(A x^{2}+B x+C\right) d x
$$

Maximum gray level difference pixel and number are marked as $f_{0}$ and 0 , contiguous pixel beside the pixelare marked as $-2,-1,1$ and 2 , the corresponding value are $f_{-2}, f_{-1}, f_{1}$ and $f_{2}$. According to the Equ.5 the output gray level difference can be described as:

$$
\begin{aligned}
& f_{-2}=\int_{n-0.5}^{n+0.5}\left(A x^{2}+B x+C\right) d x=\frac{49}{12} A-2 B+C \\
& f_{-1}=\frac{13}{12} A-B+C \\
& f_{0}=\frac{1}{12} A+C \\
& f_{1}=\frac{13}{12} A+B+C \\
& f_{2}=\frac{49}{12} A+2 B+C
\end{aligned}
$$

Simultaneous equations made by Equ. 6 Equ.10 can be used to acquire the vertex coordinate of parabola by least square method. With inverse logarithm, the subpixel edge value can be represented as:

$$
x_{g}=-\frac{-0.2 \ln f_{-2}-0.1 \ln f_{-2}+0.1 \ln f_{1}-0.2 \ln f_{2}}{2\left(0.1429 \ln f_{-2}-0.0714 \ln f_{-2}-0.1429 \ln f_{0}-0.0714 \ln f_{1}+0.1429 \ln f_{2}\right)}
$$

After the edge localization and contiguous pixel along the gradient direction, subpixel edge can be acquired form Equ.11. Bresenham algorithm is convenience for the linear scan conversion in computer graphics field[11]. In this paper, the algorithm is adopted to get its contiguous pixel along the gradient direction.

\section{Bresenham algorithm}

As presented in Fig.1, red point stands for the maximum gray level difference point, black line stands for the gradient direction and blue pixel stand for the chosen contiguous pixel. Active pixel move one unit every 
frequency along the gradient direction, secondary directional movement is determined by the midpoint method [12].

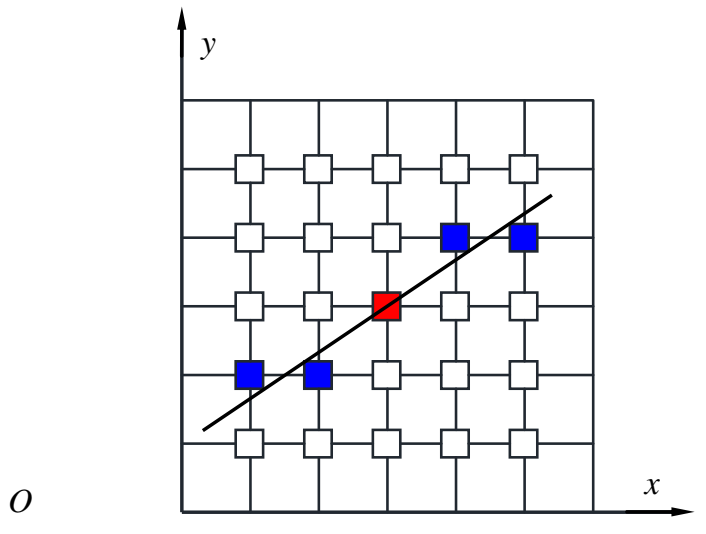

Fig. 1 Line scan conversion

Midpoint deviation discrimination

Designate the ideal line along the gradient direction, implicit function equation of the line can be described as:

$f(x, y)=y-k x-b=0$

which $k$ is the linear slope as well as the gradient, $b$ is the intercept in y direction. As presented in Fig. 2, assumed the first coordinate point is $P_{i}\left(x_{i}, y_{i}\right)$, move along the x direction, the next pixel can be chosen between $P_{a}\left(x_{i}+1, y_{i}+1\right)$ and $P_{b}\left(x_{i}+1, y_{i}\right)$. The midpoint of $P_{a}$ and $P_{b}$ is $M\left(x_{i}+1, y_{i}+0.5\right), P_{a}$ is chosen when ideal line above the midpoint $\mathrm{M}$ which means $P_{a}$ is nearer to the line compare with $P_{b}$, otherwise $P_{b}$ will be chosen as the adjacent pixel.

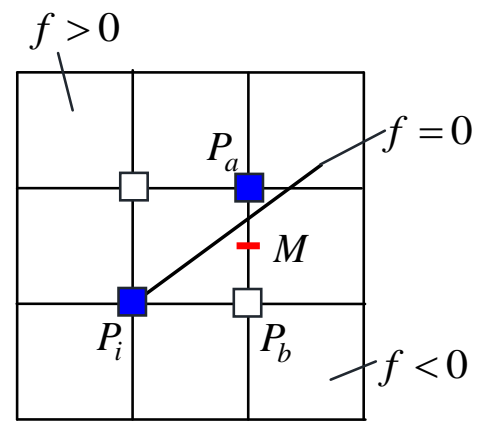

Fig. 2 Midpoint Bresenham

Midpoint deviation discrimination is integrant for the acquirement of next pixel after the first step. Substituted midpoint $\mathrm{M}$ in implicit function equation to built the midpoint deviation discrimination $d_{i}$ :

$$
d_{i}=f\left(x_{i}+1, y_{i}+0.5\right)=y_{i}+0.5-k\left(x_{i}+1\right)-b
$$

$P_{a}$ is chosen as the adjacent pixel when $d_{i}<0$ ideal line above the midpoint $\mathrm{M}$ which means active pixel move one unit along y direction. $P_{b}$ is chosen when $d_{i}>0$ ideal line below the midpoint $\mathrm{M}$ which means chosen pixel have no movement. Either $P_{a}$ or $P_{b}$ can be chosen when $d_{i}=0$ means $M$ located on the line. The deviation discrimination can also be presented as:

$$
y_{i+1}=\left\{\begin{array}{cc}
y_{i}+1, & d_{i}<0 \\
y_{i}, & d_{i} \geq 0
\end{array}\right.
$$




\section{Recurrence formulation of midpoint deviation discrimination}

Recurrence formulation is pre-requisite when midpoint deviation discrimination $d_{i}$ acquired to judge the four points on the line. As presented in Fig. 2, there are two possibility situations when considering which midpoint should be chosen to substitute the midpoint deviation discrimination and chosen the pixel after the active pixel has already have a movement along the gradient direction.

1) Midpoint coordinates of the next step is $M^{\prime}\left(x_{i}+2, y_{i}+1.5\right)$ when $d_{i}<0$, the following midpoint deviation discrimination can be prescribed as:

$$
\begin{aligned}
& d_{i+1}=f\left(x_{i}+2, y_{i}+1.5\right)=y_{i}+1.5-k\left(x_{i}+2\right)-b \\
& =y_{i}+0.5-k\left(x_{i}+1\right)-b+1-k=d_{i}+1-k
\end{aligned}
$$

2) Midpoint coordinates of the next step is $M^{\prime}\left(x_{i}+2, y_{i}+0.5\right)$ when $d_{i} \geq 0$, the following midpoint deviation discrimination can be prescribed as:

$$
\begin{aligned}
& d_{i+1}=f\left(x_{i}+2, y_{i}+0.5\right)=y_{i}+0.5-k\left(x_{i}+2\right)-b \\
& =y_{i}+0.5-k\left(x_{i}+1\right)-b-k=d_{i}-k
\end{aligned}
$$

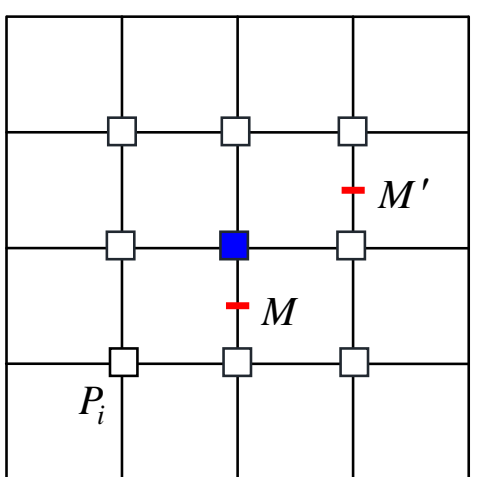

(a) $d_{i}<0$

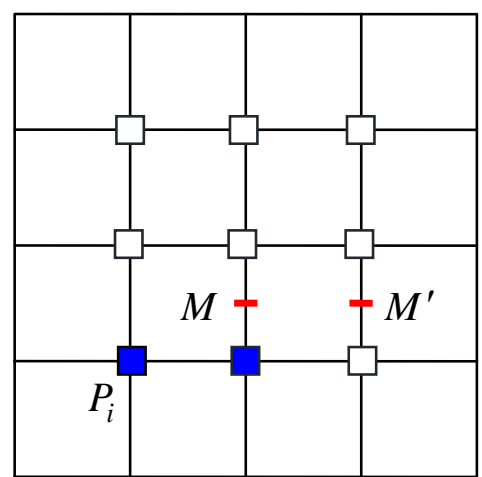

(b) $d_{i} \geq 0$

Fig. 3 Recurrence formulation of midpoint method

\section{Experimental Results}

As presented in Fig.4, An indexable insert image is used to verify the proposed edge detection method. Left image is the original image, while the right is the detected image. The result shows a good subpixel edge localization and interpolation ability. Compared with the traditional edge detection methods, the proposed method is more convenient and has a good fault tolerance for the edge roughness. Subpixel edge localization is also means a good non-contact measurement precision
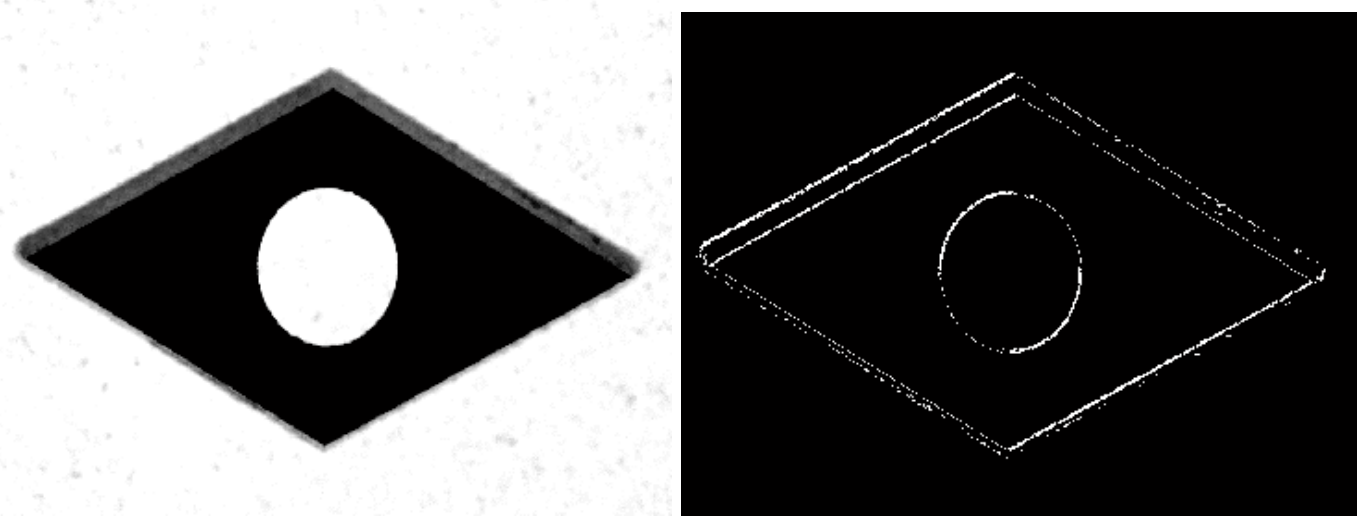

Fig. 4 Edge detection method of indexable insert 


\section{Summary}

In this paper, a new subpixel edge localization and interpolation of still image based on the Bresenham algorithm is proposed. Related research shows the maximum gray level difference is its real edge in physical space. Based on this characteristic, parameters fitting method based on the Gaussian distribution is employed to acquire the subpixel image brimpoint. Contiguous pixel is acquired according to the Bresenham algorithm. An experiment is presented to demonstrate its validity. The Results shows it is more convenient and has a good fault tolerance for the edge roughness.

\section{References}

[1] Shi Yuan. Surface profile measurement visualization of large aspheric lenses[J]. High Power Laser and Particle Beams, 2014, 26(5):141-144. DOI:10.11884/HPLPB201426.052005.

[2] Green N C, Bowen J, Hukins D W, et al. Assessment of non-contacting optical methods to measure wear and surface roughness in ceramic total disc replacements[J]. Proceedings of the Institution of Mechanical Engineers Part H Journal of Engineering in Medicine, 2015, 229.

[3] Ni Yuanmin, Wu Qian. On Improvement Segmentation Algorithm of Image Edge Contour Extraction Based on Fuzzy Morphology [J]. Journal of Southwest University (Natural Science), 2013, (12):95-100. DOI:10.3969/j.issn.1000-5471.2013.12.019.

[4] Qian Z, Wang W, Qiao T. An Edge Detection Method in DCT Domain[J]. Procedia Engineering, 2012, 29(4):344-348.

[5] Shan Y, Boon G W. Sub-pixel location of edges with non-uniform blurring: a finite closed-form approach[J]. Image \& Vision Computing, 2000, 18(13):1015-1023.

[6] Frederic Truchetet, Laligant O. Subpixel edge detection for dimensional control by artificial vision.[J]. Machine Vision Applications in Industrial Inspection VIII, 2000, 10(1):234-239.

[7] Magnan P. Detection Of Visible Photons In Ccd And Cmos: A Comparative View[J]. Nuclear Instruments \& Methods in Physics Research, 2003, 504:199-212.

[8] Ye J, Fu G, Poudel U P. High-accuracy edge detection with Blurred Edge Model[J]. Image \& Vision Computing, 2005, 23(5):453-467.

[9] Wei G X, Guo C S. Noniterative real-time coherent diffraction imaging by period sampling aperture array[J]. Scientia Sinica, 2012, 42(5):452-457.

[10] K, Jensen, D, Anastassiou. Subpixel edge localization and the interpolation of still images[J]. IEEE Transactions on Image Processing:a Publication of the IEEE Signal Processing Society, 1995, 4(3):285 295.

[11] Wang H, Chang X. 3-D RAY TRACING METHOD BASED ON GRAPHIC STRUCTURE[J]. Chinese Journal of Geophysics, 2000, 43(4):568-575.

[12] Lin Xiaoxia, Zhang Shuyou. The Algorithm of Automatic Organization of Complex View Outline Information Based on Midpoint Method[J]. JOURNAL OF IMAGE AND GRAPHICS, 2008, 13(3):541-546. 